PROCEEDINGS OF THE

AMERICAN MATHEMATICAL SOCIETY

Volume 128, Number 12, Pages 3587-3593

S 0002-9939(00)05483-6

Article electronically published on June 6, 2000

\title{
ENTIRE REGULARIZATIONS OF STRONGLY CONTINUOUS GROUPS AND PRODUCTS OF ANALYTIC GENERATORS
}

\author{
IOANA CIORANESCU AND VALENTIN KEYANTUO
}

(Communicated by Dale Alspach)

\begin{abstract}
Using entire regularizations of $C_{0}$-groups, we give a characterization of their analytic generators which we apply to the study of products of such generators.
\end{abstract}

\section{Preliminaries}

Let $X$ be a complex Banach space and $\mathcal{L}(X)$ the space of bounded linear operators on $X$. Let $A$ be a closed linear operator on $X$ with dense domain $D(A)$; let $R(A)$ denote the range, $N(A)$ the kernel of $A$, and $\rho(A)$ and $\sigma(A)$ the resolvent set and the spectrum of $A$. Throughout, given two linear operators $A$ and $B$ on $X$, by $A+B$ we denote the operator $(A+B) x=A x+B x$ with $D(A+B)=D(A) \cap D(B)$ and by $A B$ the operator $(A B) x=A(B x)$ with $D(A B)=\{x \in D(B) ; B x \in D(A)\}$.

We briefly recall basic facts concerning the concept of analytic generator for strongly continuous groups of operators, introduced by Cioranescu-Zsido 2] and used extensively in the Tomita-Takesaki theory (see [10]).

Let $U($.$) be a strongly continuous group in X$. For $\alpha \in \mathbf{C}$, we denote by $\Omega_{\alpha}$ the vertical strip

$$
\Omega_{\alpha}=\left\{\begin{array}{l}
\{z \in \mathbf{C}, 0<\operatorname{Re}(z)<\operatorname{Re}(\alpha)\} \text { if } \operatorname{Re}(\alpha)>0, \\
\{z \in \mathbf{C}, \operatorname{Re}(\alpha)<\operatorname{Re}(z)<0\} \text { if } \operatorname{Re}(\alpha)<0 .
\end{array}\right.
$$

Then for $\alpha \in \mathbf{C}$, the operator $B_{\alpha}$ is defined by

$$
\left\{\begin{array}{l}
D\left(B_{\alpha}\right)=\left\{x \in X, \exists F_{x} \in \mathcal{H} \text { ol }\left(\Omega_{\alpha}\right) \cap \mathcal{C}\left(\overline{\Omega_{\alpha}}\right): U(t) x=F_{x}(i t), t \in \mathbf{R}\right\}, \\
B_{\alpha} x:=F_{x}(\alpha), x \in D\left(B_{\alpha}\right) .
\end{array}\right.
$$

Definition 1.1. The analytic generator for $U($.$) is the operator B:=B_{1}$.

The operators $B_{\alpha}$ are closed, injective and densely defined, and the set

$$
\mathcal{D}=\left\{x \in X ; \exists F_{x} \in \operatorname{Hol}(\mathbf{C}): U(t) x=F_{x}(i t), t \in \mathbf{R}\right\}
$$

is a core for $B_{\alpha}$ (see [2]).

The case where $\rho(B) \neq \emptyset$ was first studied by Zsidó [11], who connected it to the Hilbert transform. More results were obtained recently by Monniaux [6], [7],

Received by the editors October 20, 1998 and, in revised form, February 18, 1999.

2000 Mathematics Subject Classification. Primary 47D03.

(C)2000 American Mathematical Society 
who established the connection between analytic generators and the class BIP of operators in UMD-spaces.

We recall the following definition from [8].

Definition 1.2. The operator $A$ belongs to the class $B I P(X, \omega)$ where $\omega \in[0, \pi)$ if $A$ satisfies the conditions

(H1) $(-\infty, 0) \subset \rho(A), N(A)=\{0\}, \overline{R(A)}=X$ and $\left\|t(t+A)^{-1}\right\| \leq M$ for some constant $M \geq 1$ and all $t>0$.

(H2) For all $t \in \mathbf{R}, A^{i t} \in \mathcal{L}(X)$ and there exists $K \geq 1$ such that $\left\|A^{i t}\right\| \leq$ $K e^{\omega|t|}, t \in \mathbf{R}$.

We further introduce the class UMD of Banach spaces with the unconditional martingale difference property (see Bourgain [1]).

Consider the following family of operators:

$$
H_{\varepsilon} f(t)=\frac{i}{\pi} \int_{\varepsilon \leq|t|} f(t-s) \frac{d s}{s}
$$

for $\varepsilon>0, \quad t \in \mathbf{R}$ and $f \in C_{0}^{\infty}(\mathbf{R}, X)$. Then $H f(t)=\lim _{\varepsilon \rightarrow 0} H_{\varepsilon} f(t)$ is called the Hilbert transform.

Definition 1.3. If the operator $H$ admits a continuous extension to $L^{p}(X), p \in$ $(1, \infty)$, then we say that $X$ is a UMD space.

The next result is due to S. Monniaux.

Lemma 1.4. Let $X$ be a UMD-space and $\omega \in[0, \pi)$; then $B \in B I P(X, \omega)$ if and only if $B$ is the analytic generator of a group of type $\leq \omega$.

In general Banach spaces, a similar result is not known. We conjecture that if $B$ is the analytic generator for a group of type less than $\pi$ and $\rho(B) \neq \emptyset$, then $B \in B I P(X, \pi)$.

Products of analytic generators were studied in UMD-spaces (via the sum of such operators) by Prüss-Sohr [8] and in general Banach spaces (via functional calculus) by Uiterdijk [9] (see also Monniaux [6], [7] for a particular case). In this note, we study such products using a representation formula for the analytic generator obtained by means of entire regularizations of strongly continuous groups which may present interest in itself.

\section{MAin Results}

We recall the notion of an entire $C$-group due to deLaubenfels [4], [5].

Definition 2.1. Let $C \in \mathcal{L}(X)$ be injective. The family of operators $(W(z))_{z \in \mathbf{C}}$ is an entire $C$-group if

i) $W(z)$ is an entire $\mathcal{L}(X)$-valued function of $z$;

ii) $W(z) W(\rho)=C W(z+\rho)$ for all $z, \rho \in \mathbf{C}$;

iii) $W(0)=C$.

The generator of $(W(z))$ is the operator $A$ defined as follows:

$$
\begin{gathered}
D(A):=\left\{x \in X, \lim _{t \rightarrow 0} \frac{W(t) x-C x}{t} \text { exists and is in } R(C)\right\}, \\
A x=C^{-1} \lim _{t \rightarrow 0} \frac{W(t) x-C x}{t} \text { for } x \in D(A) .
\end{gathered}
$$

We will make use of the following lemma (see also deLaubenfels [4]). 
Lemma 2.2. Let $(T(t))$ be a strongly continuous analytic semigroup with generator $A$. Then for any $t_{0}>0, T\left(t_{0}\right)$ is injective and has dense range.

Proof. We first show that $T\left(t_{0}\right)$ is one-to-one. Assume that $T\left(t_{0}\right) x=0$ for some $x \in X$. Since $t_{0}>0, \quad T\left(t_{0}\right)(X) \subset \bigcap_{n \in \mathbf{N}} D\left(A^{n}\right)$ and $\left.\frac{d^{n}}{d t^{n}} T(t) x\right|_{t=t_{0}}=A^{n} T\left(t_{0}\right) x$. Using analyticity, one obtains that $T(t) x=0$ for $0<t<t_{0}$. Strong continuity at 0 now gives $x=0$.

To show that $T\left(t_{0}\right)$ has dense range, assume that $\varphi \in X^{*}$ is such that $\left\langle\varphi, T\left(t_{0}\right) x\right\rangle$ $=0$ for every $x \in X$. By considering the function $f$ defined by $f(t)=\langle\varphi, T(t) x\rangle$ for $t>0$, and using analyticity and strong continuity of $(T(t))$ as above, one obtains $f(0)=\lim _{t \rightarrow 0}\langle\varphi, T(t) x\rangle=0=\langle\varphi, x\rangle$ for every $x \in X$ so that $\varphi=0$.

Remark. From the lemma, one can deduce the following stronger statement using an abstract Mittag-Leffler argument.

$$
\mathcal{A}:=\bigcap_{t>0} T(t)(X) \text { is dense in } X .
$$

It is also easy to see that $\mathcal{A}$ is contained in the set of entire vectors for $A$.

Proposition 2.3. Let $U($.$) be a strongly continuous group of operators on X$. Then there exists an injective operator $C$ with dense range such that $W(t)=C U(t), t \in R$, extends to an entire $C$-group $(W(z))_{z \in \mathbf{C}}$.

Proof. Define $T(t) x=\frac{1}{2 \sqrt{\pi t}} \int_{-\infty}^{\infty} e^{-\frac{S^{2}}{4 t}} U(s) x d s, t>0 ; x \in X$. Since it is wellknown that $T($.) is a strongly continuous analytic semigroup, it follows from Lemma 2.2 that

$$
C=T(1)=\frac{1}{2 \sqrt{\pi}} \int_{-\infty}^{\infty} e^{-\frac{S^{2}}{4}} U(s) d s
$$

is a bounded injective operator with dense range. Moreover $C$ commutes with $U($.$) .$ We define further $W(t)=C U(t), t \in R$. We have

$$
\begin{aligned}
W(t) x & =C U(t) x=U(t) C x=\frac{1}{2 \sqrt{\pi}} \int_{-\infty}^{\infty} e^{-\frac{S^{2}}{4}} U(s) U(t) x d s \\
& =\frac{1}{2 \sqrt{\pi}} \int_{-\infty}^{\infty} e^{-\frac{S^{2}}{4}} U(s+t) x d s=\frac{1}{2 \sqrt{\pi}} \int_{-\infty}^{\infty} e^{-\frac{(s-t)^{2}}{4}} U(s) x d s, x \in X .
\end{aligned}
$$

It is clear from this representation that we may extend $(W(t))_{t \in R}$ to $(W(z))_{x \in \mathbf{C}}$ through

$$
W(z) x=\frac{1}{2 \sqrt{\pi}} \int_{-\infty}^{\infty} e^{-\frac{(s-z)^{2}}{4}} U(s) x d s, z \in \mathbf{C}, x \in X .
$$

One can easily see that $(W(z))_{z \in \mathbf{C}}$ is an entire $C$-group.

Definition 2.4. An entire $C$-group $W($.$) such that W(t)=C U(t), t \in \mathbf{R}$, is called a $C$-entire regularization of $U($.$) .$

Remark. (i) We mention that in 4] deLaubenfels established the existence of an entire $C$-regularization for uniformly bounded groups using a certain functional calculus. 
(ii) The regularization process works as well if instead we consider strongly continuous cosine functions. In that case the regularizing operator is given by (2-1) with $U(t)$ replaced by the cosine function. We do not detail that here.

Theorem 2.5. Let $U($.$) and V($.$) be two commuting strongly continuous groups of$ operators in $X$. Then there exists an injective operator $C$ with dense range such that

$$
W(t)=C U(t) \text { and } S(t)=C V(t), t \in \mathbf{R} \text {, extend to entire } C \text {-groups. }
$$

Proof. Let

$$
C_{1} x=\frac{1}{2 \sqrt{\pi}} \int_{-\infty}^{\infty} e^{-\frac{s^{2}}{4}} U(s) x d s, C_{2} x=\frac{1}{2 \sqrt{\pi}} \int_{-\infty}^{\infty} e^{\frac{-s^{2}}{4}} V(s) x d s, x \in X .
$$

Then the operator $C=C_{1} C_{2}$ has all the required properties.

Theorem 2.6. Let $U($.$) be a strongly continuous group in X$ and $W($.$) an entire C$ regularization of $U($.$) . Then the analytic generator B$ of $U($.$) has the representation$

$$
B=C^{-1} W(-i)
$$

Proof. We first note that the operator $C^{-1} W(-i)$ is closed. Moreover, from

$$
U(t)=C^{-1} W(t), t \in \mathbf{R},
$$

we obtain

$$
B x=C^{-1} W(-i) x, x \in \mathcal{D}
$$

where $\mathcal{D}$ is defined by $(\underline{1-2})$.

In fact, for $x \in \mathcal{D}$, it follows from the defintion of $\mathcal{D}$ that the mapping $t \mapsto$ $U(t) x$ has an entire extension, namely $F(i z)$; moreover, $W(z) x=C F_{x}(i z)$ so that $x \in D\left(C^{-1} W(z)\right.$ for every $z \in \mathbf{C}$. Therefore, $\mathcal{D} \subset D\left(C^{-1} W(z)\right)$. This fact may also be deduced from 5, Proposition 7.7(3) and Theorem 7.8].

Since $\mathcal{D}$ is a core for $B$ it follows that $B \subset C^{-1} W(-i)$. In order to prove (2-2), it suffices to prove that $\mathcal{D}$ is a core for $C^{-1} W(-i)$.

Let $x \in D\left(C^{-1} W(-i)\right)$. By (2-3) we have

$$
C W(-i+t) x=W(t) W(-i) x=C U(t) W(-i) x, t \in \mathbf{R},
$$

i.e.

$$
W(-i+t) x=U(t) W(-i) x, t \in \mathbf{R} .
$$

Since $W(-i) x=C y$, for some $y \in X$, we obtain

$$
W(-i+t) x=C U(t) y \in R(C) .
$$

Define further for $n \in \mathbf{N}$

$$
x_{n}=\sqrt{\frac{n}{\pi}} \int_{-\infty}^{\infty} e^{-n t^{2}} U(t) x d t .
$$


Then $x_{n} \in \mathcal{D}$ and $\lim _{n \rightarrow \infty} x_{n}=x$. We have by (2-4)

$$
\begin{aligned}
W(-i) x_{n} & =\sqrt{\frac{n}{\pi}} \int_{-\infty}^{\infty} e^{-n t^{2}} W(-i) U(t) x d t \\
& =\sqrt{\frac{n}{\pi}} \int_{-\infty}^{\infty} e^{-n t^{2}} C^{-1} W(-i) W(t) x d t \\
& =\sqrt{\frac{n}{\pi}} \int_{-\infty}^{\infty} e^{-n t^{2}} W(-i+t) x d t \\
& =C\left(\sqrt{\frac{n}{\pi}} \int_{-\infty}^{\infty} e^{-n t^{2}} U(t) y d t\right) .
\end{aligned}
$$

Consequently, $W(-i) x_{n} \in R(C)$ and

$$
\lim _{n} C^{-1} W(-i) x_{n}=\lim _{n} \sqrt{\frac{n}{\pi}} \int_{-\infty}^{\infty} e^{-n t^{2}} U(t) y d t=y=C^{-1} W(-i) x .
$$

Hence $\mathcal{D}$ is a core for $C^{-1} W(-i)$ and this completes the proof.

Remark. (i) By the same method, we can prove that $B_{\alpha}=C^{-1} W(-i \alpha), \alpha \in \mathbf{C}$.

(ii) If $x \in \mathcal{D}$ and $B$ is the analytic generator of the strongly continuous group with infinitesimal generator $G$, then

$$
B x=\sum_{n=0}^{\infty} \frac{(-i)^{n}}{n !} G^{n} x .
$$

In fact, for $x \in \mathcal{D}, W(t) x=C U(t) x, t \in \mathbf{R}$, has an entire extension and since $\mathcal{D} \subset D\left(C^{-1} W(t)\right)$, we may write

$$
U(t) x=C^{-1} W(t) x=\sum_{n=0}^{\infty} \frac{(t)^{n}}{n !} G^{n} x
$$

It then suffices to write this formula for $z \in \mathbf{C}$ and substitute $-i$ for $z$.

Proposition 2.7. Let $U($.$) and V($.$) be two commuting strongly continuous groups$ with analytic generators $A$ and $B$. Then $A+B$ and $A B$ are closable.

Remark. By Theorem $[2.5$ there is an injective operator $C \in \mathcal{L}(X)$ defining two $C$-entire regularizations $W($.$) and S($.$) of U($.$) and V($.$) , respectively. Then by the$ above theorem

$$
A=C^{-1} W(-i), \quad B=C^{-1} S(-i) .
$$

It follows that

$$
\begin{gathered}
A+B=C^{-1} W(-i)+C^{-1} S(-i) \subset C^{-1}(W(-i)+S(-i)), \\
A B=C^{-1} W(-i) C^{-1} S(-i) \subset C^{-2} W(-i) S(-i)
\end{gathered}
$$

and this yields the result.

As an application of the above closability result, we give a new proof of the following result obtained by M. Uiterdijk [9, Theorem 3.4.12].

Theorem 2.8. Let $U($.$) and V($.$) be two commuting strongly continuous groups$ with analytic generators $A$ and $B$ respectively. Then $\overline{A B}$ is the analytic generator of the group $U() V.($.$) .$ 
Proof. Using the same notations as in the proof of the foregoing proposition, we note that $A=C^{-1} W(-i), B=C^{-1} S(-i)$ and that the operator $K=C^{-2} W(-i) S(-i)$ is the analytic generator of the group $U() V.($.$) . Moreover, A B$ is closable and (2-6) holds. For $x \in X$ and $n \in \mathbf{N}$, define

$$
x_{n}=\frac{n^{2}}{\pi} \int_{-\infty}^{\infty} \int_{-\infty}^{\infty} e^{-n^{2}\left(t^{2}+s^{2}\right)} U(t) V(s) x d s d t .
$$

Then since $x_{n}$ is an entire vector for the group $U() V.(),. \quad x_{n} \in D(K)$; it is furthermore clear that $\lim _{n} x_{n}=x$.

We shall prove that $x_{n} \in D(A B)$. In fact, we have

$$
\begin{aligned}
S(-i) x_{n} & =\frac{n^{2}}{\pi} \int_{-\infty}^{\infty} e^{-n^{2} s^{2}} S(-i) V(s)\left(\int_{-\infty}^{\infty} e^{-n^{2} t^{2}} U(t) x d t\right) d s \\
& =\frac{n^{2}}{\pi} \int_{-\infty}^{\infty} e^{-n^{2} s^{2}} S(-i+s)\left(\int_{-\infty}^{\infty} e^{-n^{2} t^{2}} U(t) x d t\right) d s \\
& =\frac{n^{2}}{\pi} \int_{-\infty}^{\infty} e^{-n^{2}(i+u)^{2}} S(u)\left(\int_{-\infty}^{\infty} e^{-n^{2} t^{2}} U(t) x d t\right) d u \\
& =C\left[\frac{n^{2}}{\pi} \int_{-\infty}^{\infty} e^{-n^{2}(i+u)^{2}} V(u)\left(\int_{-\infty}^{\infty} e^{-n^{2} t^{2}} U(t) x d t\right) d u\right]
\end{aligned}
$$

Consequently, $S(-i) x_{n} \in R(C)$; that is, $x_{n} \in D(B)$ and

$$
\begin{aligned}
W(-i) B x_{n} & =\frac{n^{2}}{\pi} \int_{-\infty}^{\infty} e^{-n^{2}(i+u)^{2}} V(u)\left(\int_{-\infty}^{\infty} e^{-n^{2} t^{2}} W(-i) U(t) x d t\right) d u \\
& =\frac{n^{2}}{\pi} \int_{-\infty}^{\infty} e^{-n^{2}(i+u)^{2}} V(u)\left(\int_{-\infty}^{\infty} e^{-n^{2} t^{2}} W(-i+t) x d t\right) d u \\
& =\frac{n^{2}}{\pi} \int_{-\infty}^{\infty} e^{-n^{2}(i+u)^{2}} V(u)\left(\int_{-\infty}^{\infty} e^{-n^{2}(i+r)^{2}} W(r) x d r\right) d u \\
& =C\left[\frac{n^{2}}{\pi} \int_{-\infty}^{\infty} e^{-n^{2}(i+u)^{2}} V(u)\left(\int_{-\infty}^{\infty} e^{-n^{2}(i+r)^{2}} U(r) x d r\right) d u\right] .
\end{aligned}
$$

It follows that $B x_{n} \in D(A)$ and

$$
\begin{aligned}
A B x_{n} & =\frac{n^{2}}{\pi} \int_{-\infty}^{\infty} \int_{-\infty}^{\infty} e^{-n^{2}\left[(i+u)^{2}+(i+r)^{2}\right]} U(r) V(u) x d r d u \\
& =K x_{n} .
\end{aligned}
$$

Suppose finally that $x \in D(K)$. Then we have

$$
\begin{aligned}
W(-i) S(-i) x_{n} & =\frac{n^{2}}{\pi} \int_{-\infty}^{\infty} \int_{-\infty}^{\infty} e^{-n^{2}\left(t^{2}+s^{2}\right)} U(t) V(s) W(-i) S(-i) x d s d t \\
& =C^{2}\left[\frac{n^{2}}{\pi} \int_{-\infty}^{\infty} \int_{-\infty}^{\infty} e^{-n^{2}\left(t^{2}+s^{2}\right)} U(t) V(s) K x d s d t\right] .
\end{aligned}
$$

It follows that

$$
A B x_{n}=K x_{n}=\frac{n^{2}}{\pi} \int_{-\infty}^{\infty} \int_{-\infty}^{\infty} e^{-n^{2}\left(t^{2}+s^{2}\right)} U(t) V(s) K x d s d t .
$$

Hence $\lim _{n} A B x_{n}=K x$ and the proof is complete. 
Remark. To justify the change of variable used in the proof of Theorem 2.8, we note that $z \mapsto \int_{-\infty}^{\infty} e^{-n^{2} s^{2}} W(s+z) d s$ and $z \mapsto \int_{-\infty}^{\infty} e^{-n^{2}(s-z)^{2}} W(s) d s$ are two entire functions which coincide when $z \in \mathbf{R}$.

As a direct consequence of Lemma 1.4, we have the following result of Prüss and Sohr [8]:

Corollary 2.9. Let $X$ be a $U M D$ space, $A \in B I P\left(X, w_{A}\right), B \in B I P\left(X, \omega_{B}\right)$. Assume that $A$ and $B$ are resolvent commuting; that is, $(\lambda-A)^{-1}(\mu-B)^{-1}=$ $(\mu-B)^{-1}(\lambda-A)^{-1}$ for $\lambda \in \rho(A), \mu \in \rho(B)$ and $\omega_{A}+\omega_{B}<\pi$. Then $A B$ is closable and $\overline{A B} \in B I P\left(X, \omega_{A}+\omega_{B}\right)$.

\section{ACKNOWLEDGMENT}

We would like to thank the referee for several useful suggestions.

\section{REFERENCES}

[1] Bourgain J., Some remarks on Banach spaces in which martingale differences are unconditional, Ark. Math. 22 (1983), 163-168. MR 85a:46011

[2] Cioranescu I., Zsidó L., Analytic generators for one-parameter groups of operators, Tôhoku Math. J. 28 (1976), 327-362. MR 55:3872

[3] Cioranescu I., Zsidó L., Analytic generators for one-parameter cosine families, Acta Sci. Math. 45 (1983), 95-109. MR 85c:47043

[4] de Laubenfels R., Entire solutions of the abstract Cauchy problem, Semigroup Forum 42 (1991), 83-105. MR 91i:47055

[5] de Laubenfels R., "Existence Families, Functional Calculi and Evolution Equations", Lecture Notes in Math., 1570, Springer-Verlag, 1994. MR 96b:47047

[6] Monniaux S., "Générateur analytique et classe d'opérateurs BIP", Thèse, Besançon 1995.

[7] Monniaux S., A new approach to the Dore-Venni theorem, Math. Nachrichten 204 (1999), 163-183. CMP 99:16

[8] Prüss J. and Sohr H., On operators with bounded imaginary powers in Banach spaces, Math. Z. 203 (1990), 429-452. MR 91b:47030

[9] Uiterdijk, M., "Functional Calculi for Closed Operators", Ph.D. Thesis, Delft 1998.

[10] Zsidó L., Analytic generators and the foundation of the Tomita-Takesaki theory, Proc. Int. School of Math. Phys. Univ. Camerino, (1974), 182-267.

[11] Zsidó L., Spectral properties of the analytic generator and singular integrals, Mem. Acad. Lincei, 17 (1983), 105-134. MR 87f:47062

Department of Mathematics and Computer Science, University of Puerto Rico, Box 23355, San Juan, Puerto Rico 00931-3355

E-mail address: eciorane@rrpac.upr.clu.edu

Department of Mathematics and Computer Science, University of Puerto Rico, Box 23355, San Juan, Puerto Rico 00931-3355

E-mail address: keyantuo@rrpac.upr.clu.edu 